\title{
Retinal detachment following anterior chamber lens implantation for the correction of ultra-high myopia in phakic eyes
}

\author{
A J E Foss, P H Rosen, R J Cooling
}

\begin{abstract}
The development of retinal detachment is reported in three eyes, of two patients, following implantation of an anterior chamber intraocular lens (AC-IOL) in phakic eyes for the correction of ultra-high myopia. The presence of an AC-IOL may hamper the identification of retinal breaks and removal of the intraocular lens may prove necessary to improve visualisation. Shallowing of the anterior chamber may also occur during, or following, vitreoretinal surgery with risk of endothelial contact. The risks and relative contraindications of $\mathrm{AC}-\mathrm{IOL}$ implantation into phakic eyes are discussed. (Brf Ophthalmol 1993; 77: 212-213)
\end{abstract}

A negatively powered anterior chamber intraocular lens (AC-IOL) implant positioned in front of the crystalline lens can be employed as a treatment for high myopia. Procedures for the correction of myopia including excimer laser photoablation $^{1}$ or radial keratotomy ${ }^{2}$ are not effective for the correction of ultra-high myopia for which clear lens extraction or AC-IOL implantation has been advocated. ${ }^{3-6}$ The theoretical advantages of AC-IOL implantation are a more controlled final refraction and preservation of accommodation.

Three eyes, of two patients, which developed retinal detachment following this procedure are reported.

Accepted for publication

9 December 1992

Moorields Eye Hospital, City Road, London

A J E Foss

R J Cooling

Correspondence to:

R J Cooling, Moorfields Eye Hospital, City Road, London ECIV 2PD.

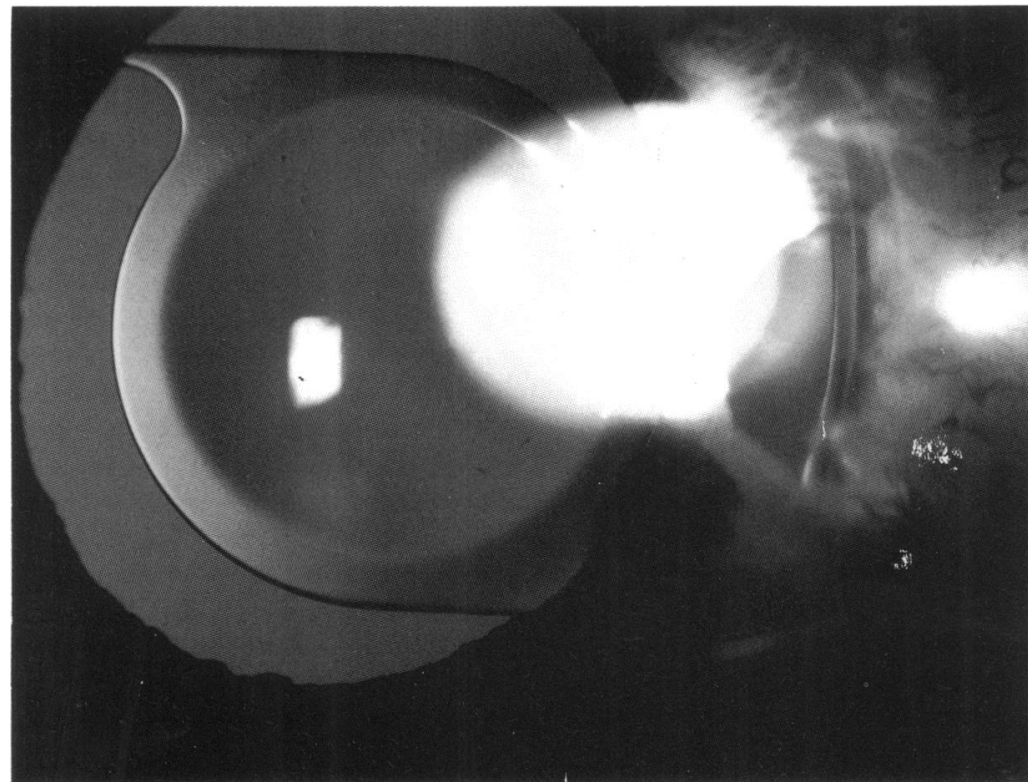

Figure 1 Retroillumination photograph showing a ZB-5M anterior chamber lens implanted in front of a clear crystalline lens.
Case 1

A 25-year-old man had a spectacle correction of $-20 \mathrm{DS} /+2 \times 90$ in both eyes with a corrected acuity of $6 / 12$ right and $6 / 18$ left. Bilateral ZB-5M anterior chamber lenses (Dormilens, France) were implanted to give corrected visual acuities of $6 / 12$ in both eyes. Three months after the left eye was implanted, he was referred with a 5 day history of progressive loss of vision in that eye.

On examination, visual acuities were $6 / 12$ right and 2/60 left. There was a left superotemporal retinal detachment involving the macula, due to three $U$-shaped tears in relation to the vitreous base.

Conventional retinal detachment surgery was performed with combined drainage of subretinal fluid, cryotherapy, and a segmental circumferential explant. The retina was successfully reattached and remained flat after 7 months with recovery of $6 / 12$ vision.

\section{Case 2}

A 30-year-old man had a -28 DS spectacle correction with a visual acuity of $6 / 18$ in each eye. Bilateral AC-IOLs were implanted. The left eye was implanted with a ZB-5M anterior chamber lens (Fig 1) which gave a spectacle corrected visual acuity of $6 / 9$.

He was referred 13 months after surgery with a 2 week history of loss of vision in the left eye due to a subtotal retinal detachment. No break could be visualised preoperatively as the implant significantly impaired the view of the retina.

He underwent pars plana vitrectomy and an internal search. A break was identified posterior to the equator and adjacent to the superonasal vascular arcade. Internal drainage and fluid/gas exchange were performed with endolaser demarcation of the retinal break.

Although the retina was initially reattached, recurrent detachment occurred on three occasions when no break could te visualised preoperatively: on the first occasion, a posterior break was identified inferotemporally after an internal search and the retina was reattached with gas tamponade. On the second occasion, no break could be visualised preoperatively or following an internal search. The AC-IOL was removed and the crystalline lens removed by pars plana ultrasonic lensectomy. A posterior break was then identified superonasally and the retina was reattached with silicone oil and endolaser applied to the break. On the third occasion, redetachment occurred inferiorly due to two new inferior atrophic breaks associated with proliferative vitreoretinopathy. These were success- 
fully closed with an inferior explant and a further injection of silicone oil. The retina has remained flat but the visual acuity has not improved beyond hand movements.

The right eye was initially implanted with a ZB lens but the lens was noted to be undersized and 1 year later was exchanged for a larger diameter ZB-5M lens. Vision did not recover postoperatively and he was again referred to the vitreoretinal unit with a visual acuity of hand movements in the right eye and a subtotal retinal detachment.

The right eye underwent vitrectomy and internal search and a break was identified close to the inferonasal vascular arcade. A fluid/gas exchange was performed and endolaser applied to the break with reattachment of the retina. The postoperative period was complicated by shallowing of the anterior chamber, bringing the implant into close proximity to the corneal endothelium. The anterior chamber reformed as the gas reabsorbed and the vision returned to $6 / 36$. The cornea has subsequently developed central corneal guttata.

\section{Discussion}

The implantation of negatively powered anterior AC-IOL into phakic eyes to correct high myopia was first performed in the 1950s but the procedure was abandoned owing to a high complication rate. The idea has been revived, mainly by Baikoff with his ZB and ZB-5M lenses based on the Kelmann AC-IOL, ${ }^{3}$ and by Worst in Holland with the lobster claw lens. ${ }^{78}$ Our patients were implanted with either the ZB or the ZB-5M lenses.

There is a well-recognised association between myopia and retinal detachment ${ }^{9}$ but there is no reported incidence, or relative risk of detachment for the ultra-high myope (over -15 DS). Similarly, the incidence of retinal detachment following AC-IOL implantation in phakic eyes is not known. The largest series reported by Barraquer in $1959^{4}$ and Baikoff in $1991^{3}$ described two detachments after 236 implantations and one after 163 implantations respectively. The other two published series are much smaller - Praeger reporting on nine implant procedures ${ }^{5}$ and Dvali on eight, ${ }^{6}$ and neither author reported retinal detachment. These studies, however, failed to state the length of follow up.

Retinal detachment is not uncommon in such highly myopic eyes and may present a difficult management problem. The breaks may be difficult to identify in the highly myopic fundus and visualisation is further hampered by the presence of the AC-IOL with its small $(4.5 \mathrm{~mm})$ optic. Furthermore, some of these patients may have a predisposing vitreoretinal degeneration such as Stickler's syndrome in which retinal detachments have a relatively poor prognosis.

If the breaks can be visualised and are anterior, then the detachment can be managed successfully with conventional scleral buckling procedures as demonstrated in the first case. Failure to visualise the breaks or breaks posterior to the equator may necessitate an internal approach. Removal of the AC-IOL (possibly combined with clear lens extraction to correct the myopia) should be considered to improve the view if the break(s) are not identified following internal search.

This report has focused on the retinal complications, but the other major concern is progressive damage to the corneal endothelium. The optics, being negatively powered, are progressively thicker (and the anterior chamber shallower) towards the periphery and this limits the size of the optic. Damage to the corneal endothelium has been observed by specular microscopy with the ZB lens ${ }^{1011}$ and progressive endothelial damage in $4 \%$ of patients implanted with the Worst lobster claw lens. ${ }^{7}$ Indentation microsurgery ${ }^{12}$ and the use of intraocular gas tamponade, during detachment surgery, can cause transient shallowing of the anterior chamber and progressive endothelial damage in $4 \%$ of patients implanted with the Worst lobster claw lens. ${ }^{7}$ Indentation microsurgery ${ }^{12}$ and the use of intraocular gas tamponade during detachment surgery can cause transient shallowing of the anterior chamber and endothelial touch. The right eye of the second patient had evidence of endothelial damage but the exact mechanism for this was not clear.

AC-IOL implantation in such highly myopic eyes renders subsequent retinal detachment surgery more difficult. We recommend that these patients should have complete fundus examination before surgery of this nature. It should be noted that in eyes with lattice degeneration, one third of retinal breaks occur in normal retinas, ${ }^{13}$ and prophylaxis does not give complete protection. ${ }^{14}$ The presence of vitreoretinal degenerations such as Stickler's syndrome should be considered a contraindication to implantation, especially while the procedure remains under evaluation.

We thank Mr Peter Choyce for allowing us to report on patients in his care.

1 Waring GO, Lynn MJ, Nizam A, Kutney MH, Courden JW, Culbertson W, et al. Results of the prospective evaluation of radial keratotomy (PERK) study five years after surgery. Ophthalmology 1991; 98: 1164-76.

2 Gartry DS, Kerrmuir MG, Marshall J. Excimer laser photorefractive keratectomy: eighteen months follow up. Ophthalmology 1992; 99: 1209-19.

3 Baikoff G. Phakic anterior chamber intraocular lenses. Int Ophthalmol Clin Refract Surg 1991; 31: 75-86.

4 Barraquer J. Anterior chamber plastic lenses. Results of and conclusions from five years' experience. Trans Ophthalmol Soc UK 1959; 79: 393-424.

5 Praeger DL. Innovations and creativity in contemporary ophthalmology: preliminary experience with the phakic ophthalmology: preliminary experience with the phakic myopic intraocular lens. Ann Ophthalmol 1988; 20: 456-62. pvali ML. Correction of high myopia by means of an extra-
pupillary iris-lens. (Preliminary communication.) Russ Ann

7 Fechner PU, Strobel J, Winchmann W. Correction of myopia by implantation of a concave Worst-Iris claw lens into phakic eyes. Refract Corneal Surg 1991; 7: 286-98.

8 Fechner PU, van der Heijde GL, Worst JGF. The correction of myopia by lens implantation into phakic eyes. Am $\mathcal{J}$ Ophthalmol 1989; 107: 659-63.

9 Schepens CL, Marden D. Data on the natural history of retina detachment: further characterization of certain unilateral non traumatic cases. Am $\mathcal{F}$ Ophthalmol 1966; 61: 213-26.

10 Mimouni F, Colin J, Koff V, Bonnet P. Damage to the corneal endothelium from anterior chamber intraocular lenses in phakic myopic eyes. Refract Corneal Surg 1991; 7: 277-81.

11 Saragoussi JJ, Cotinat J, Renard G, Savoldelli M, Abenhaim A, Pouliquen Y. Damage to the corneal endothelium by A, Pouliquen Y. Damage to the corneal endothelium by minus power anterior cham
Corneal Surg 1991; 7: 282-5.

12 Rosen PH, Wong HC, McLeod D. Indentation microsurgery: internal searching for retinal breaks. Eye 1989; 3: 277-81.

13 Byer NE. Lattice degeneration of the retina. Surv Ophthalmol 1979; 23: 213-48.

14 Michels RG, Wilkinson LP, Rice TA. Retinal detachment. St Louis: Mosby, 1990: 1059-109. 